\title{
On the Oriented Game Chromatic Number*
}

\author{
J. Nešetři $1^{\dagger}$ \\ Dept. of Appl. Math. and Institute of Theoretical Computer Sciences (ITI), \\ Charles University, Prague, Czech Republic \\ nesetril@kam-enterprise.ms.mff.cuni.cz \\ E. Sopena
}

LaBRI, Université Bordeaux 1, 345 cours de la Libération

33405 Talence Cedex, France

sopena@labri.u-bordeaux.fr

Submitted: March 6, 2000; Accepted: May 18, 2000.

Subject Classification: 05C15, 68R05.

Keywords: oriented graph coloring, coloring games.

\begin{abstract}
We consider the oriented version of a coloring game introduced by Bodlaender [On the complexity of some coloring games, Internat. J. Found. Comput. Sci. 2 (1991), 133-147]. We prove that every oriented path has oriented game chromatic number at most 7 (and this bound is tight), that every oriented tree has oriented game chromatic number at most 19 and that there exists a constant $t$ such that every oriented outerplanar graph has oriented game chromatic number at most $t$.
\end{abstract}

\section{Introduction}

Let $G$ be an undirected graph, with vertex set $V(G)$ and edge set $E(G)$, and $X$ be a set of colors. We consider the two-players game defined as follows. The two players are Alice and Bob and they play alternatively with Alice having the first move. Alice's goal is to provide a proper $k$-coloring of $G$ and Bob's goal is to prevent her from doing so. A move consists in choosing an uncolored vertex $u$ and assigning it a color from the set $X$ distinct from the colors previously assigned (by either player) to the neighbors of $u$. If after $|V(G)|$ moves the graph is colored then Alice wins, otherwise Bob wins. In other

*This work has been partially supported by the Barrande Grant 02887-WD and the NATO Collaborative Research Grant 97-1519.

${ }^{\dagger}$ Partially supported by the Project LN00A056 of the Czech Ministry of Education. 
words, Bob wins whenever an impass is reached before the whole graph is colored, that is if, at some intermediate step, for every uncolored vertex $u$ and every color $\alpha, u$ has some neighbour with color $\alpha$.

The game chromatic number of $G$, denoted by $g c n(G)$, is then defined as the least cardinality of a color set $X$ for which Alice has a winning strategy. This parameter is well defined since we clearly have $\chi(G) \leq \operatorname{gcn}(G) \leq|V(G)|$.

The game chromatic number of a graph was first introduced by Bodlaender in [1] and then studied by Bodlaender and Kratsch [2], Faigle, Kern, Kierstead and Trotter [7], Kierstead and Trotter [11], Dinski and Zhu [5], Guan and Zhu [8] and Zhu [17, 18]. In particular, Kierstead and Trotter proved in [11] that $\operatorname{gcn}(G) \leq 33$ for every planar graph $G$ (the existence of an upper bound was conjectured by Bodlaender). This bound was later improved by Dinski and Zhu [5] who proved that if a graph $G$ has acyclic chromatic number $a$ then $\operatorname{gcn}(G) \leq a(a+1)$. (The acyclic chromatic number of a graph $G$ is the minimum number of colors in a proper coloring of $G$ such that every cycle in $G$ uses at least three colors.) Using a result of Borodin [3], which states that every planar graph has acyclic chromatic number at most 5 , we get $\operatorname{gcn}(G) \leq 30$ for every planar graph $G$. Using a different technique (not based on acyclic colorings), Zhu recently further reduced this bound to 19 [17]. (Later, Kierstead slightly improved this technique to get a bound of 18 [9].) On the other hand, it is known that there exist planar graphs with game chromatic number 8. The edge-coloring version of this game was considered by Cai and Zhu [4] and by Lam, Shiu and Xu [12].

In this paper, we are interested in the oriented version of this game. Let $\vec{G}$ be an oriented graph, that is a digraph with no opposite arcs. A mapping $c$ from $V(\vec{G})$ to a set of colors $X$ is an oriented coloring of $\vec{G}$ if $(i) c(u) \neq c(v)$ for every arc $u v$ and (ii) $c(u)=c(x)$ implies $c(v) \neq c(w)$ for every two arcs $u v$ and $w x$ ( $v$ and $w$ not necessarily distinct). Observe that condition ( $i i)$ implies in particular that any two vertices linked by a directed 2-path, that is a directed path of length 2, must be assigned distinct colors.

The oriented chromatic number of $\vec{G}$, denoted by $\vec{\chi}(\vec{G})$, is then defined as the least cardinality of a set $X$ such that $\vec{G}$ has an oriented coloring on $X$. Raspaud and Sopena proved in [15] that if an undirected graph $G$ has acyclic chromatic number $a$ then every orientation $\vec{G}$ of $G$ has oriented chromatic number at most $a \times 2^{a-1}$. (Thanks to Borodin's result, this gives in particular that $\vec{\chi}(\vec{G}) \leq 80$ for every oriented planar graph $\vec{G}$.)

Chromatic and oriented chromatic numbers can be equivalently defined in terms of graph homomorphisms. A homomorphism $\varphi$ of a graph $G$ to a graph $H$, denoted $\varphi$ : $G \longrightarrow H$, is an edge-preserving mapping from $V(G)$ to $V(H)(\varphi(u) \varphi(v)$ is an edge in $H$ whenever $u v$ is an edge in $G$ ). Such a mapping $\varphi$ is called a $H$-coloring of $G$. The chromatic number of an undirected graph $G$ is then the least $k$ such that $G$ admits a homomorphism to the complete graph $K_{k}$ on $k$ vertices. Similarly, the oriented chromatic number of an oriented graph $\vec{G}$ is the least $k$ such that there exists an oriented target graph $\vec{H}$ on $k$ vertices such that $\vec{G}$ admits a $\vec{H}$-coloring (similarly, homomorphisms of oriented graphs are defined as arc-preserving vertex mappings).

Let $\vec{G}$ be an oriented graph and $\vec{H}$ be a fixed oriented target graph whose vertex set will be used as the set of colors. We consider the oriented version of the coloring game 
defined as follows. Again the two players are Alice and Bob and they play alternatively with Alice having the first move. Alice's goal is to provide a $\vec{H}$-coloring of $\vec{G}$ and Bob's goal is to prevent her from doing so. A move consists in choosing an uncolored vertex $u$ and assigning it a color $\alpha$ from the set $V(\vec{H})$ such that:

1. if $v$ is a vertex with color $\beta$ and $u v$ (resp. $v u$ ) is an arc in $\vec{G}$ then $\alpha \beta$ (resp. $\beta \alpha$ ) is an arc in $\vec{H}$,

2. if $w$ is a vertex with color $\gamma$ and there exists a directed 2-path linking $u$ and $w$ (in either direction) then $\alpha \neq \gamma$.

In other words, the (partial) oriented coloring of $\vec{G}$ obtained after such a move can be extended to an oriented coloring of the whole graph $\vec{G}$ (but not necessarily to a $\vec{H}$-coloring of $\vec{G}$ ). Observe that the undirected game of Bodlaender can also be defined in the same way by taking the complete graph $K_{k}$ as a target graph and dropping condition (2) above (which comes from the fact that in the oriented case the target graph has no opposite arcs).

In this oriented game, Bob's goal is thus to "surround" some uncolored vertex $u$ in such a way that either there exists no color in $\vec{H}$ which is compatible with the already colored neighbours of $u$, or such colors exist but all of them are already assigned to some vertices in $\vec{G}$ which are linked to $u$ by a directed 2 -path.

The oriented game chromatic number of $\vec{G}$, denoted by $\operatorname{ogcn}(\vec{G})$, is then defined as the least $k$ such that there exists an oriented target graph $\vec{H}$ on $k$ vertices for which Alice has a winning strategy. The fact that this number is well-defined is not as immediate as it is in the undirected case and this will be established in the next section. Since every oriented coloring of an oriented graph is indeed a coloring of the corresponding underlying undirected graph, we get that $\operatorname{gcn}(G) \leq \operatorname{ogcn}(\vec{G})$ for every orientation $\vec{G}$ of every undirected graph $G$.

Our main interest is in characterizing families of oriented graphs having bounded oriented game chromatic number. In the next section, we introduce two types of "Golike" games (by analogy to the Go board-game) and show how these games may help for proving that families of graphs have bounded (oriented or not) game chromatic number. Using that, we prove in Section 3 that the families of oriented paths, oriented cycles or oriented trees have bounded oriented game chromatic number and in Section 4 that the family of oriented outerplanar graphs has bounded oriented game chromatic number. Finally, we discuss in Section 5 another type of oriented coloring game and state some open problems.

\section{Go-type games and coloring games}

We introduce in this section two new games and show how they are related to the coloring games discussed in the previous section. For these games, we shall play with tokens instead of colors (or, equivalently, with only one color) and speak about marked or umarked vertices. 


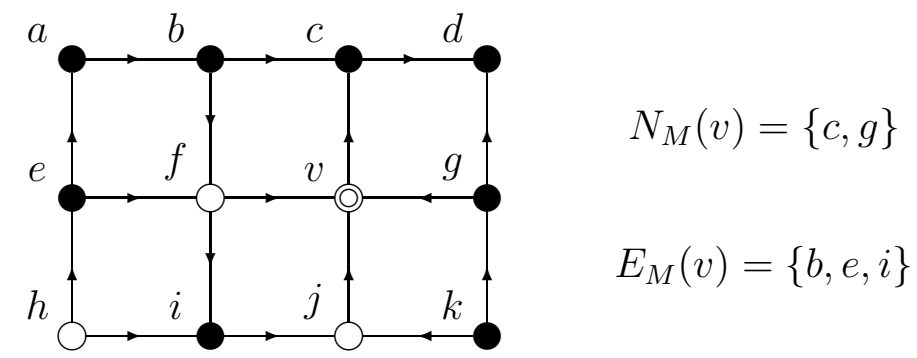

Figure 1: A vertex $v$ with extended score $s^{*}(v)=2+3=5$

Let $G$ be an undirected graph whose vertices are either marked or unmarked. We set $V(G)=M(G) \cup U(G)$ where $M(G)$ and $U(G)$ respectively denote the set of marked vertices and the set of unmarked vertices. For every unmarked vertex $u \in U(G)$, we denote by $N_{M}(u)$ the set of marked neighbours of $u$ and by $s(u)$ the cardinality of $N_{M}(u)$, called the score of $u$. The score of the graph $G$, denoted by $s(G)$, is then defined as $s(G)=\operatorname{Max}\{s(u): u \in U(G)\}$ if $U(G)$ is non-empty and $s(G)=0$ otherwise.

Let now $G$ be an undirected graph on $n$ vertices all of whose vertices are unmarked $(U(G)=V(G))$ and $k$ be a positive integer. The two-players games Go(k) is defined as follows. Alice and Bob play alternatively with Alice having the first move. A move consists in marking any unmarked vertex. The game thus finishes after $n$ moves, when all vertices are marked. Alice wins the game if after each move (of any player) the score of the current graph is at most $k$.

The Go-number of $G$, denoted by $G o(G)$, is then defined as the least $k$ such that Alice has a winning strategy in the game $G o(k)$. We clearly have $G o(G) \leq \Delta(G)$ for every graph $G$, where $\Delta(G)$ stands for the maximum degree of $G$.

It is easy to see that any winning strategy for playing the game $G o(k)$ on an undirected graph $G$ induces a winning strategy for playing the coloring game on $G$ with $k+1$ colors: considering colored vertices as marked, if every uncolored vertex has at most $k$ colored neighbours after each move then every uncolored vertex is colorable provided that we have at least $k+1$ colors available. We have proved the following

Theorem 1 For every undirected graph $G, \operatorname{gcn}(G) \leq G o(G)+1$.

A similar parameter, called the game coloring number of a graph, was considered by Zhu in [17]. The game coloring number $g \operatorname{col}(G)$ of a graph $G$ is given by $g \operatorname{col}(G)=$ $G o(G)+1$ and was used for deriving an upper bound for the game chromatic number of planar graphs.

In order to get a similar result for the oriented version of the coloring game, we now introduce a new kind of Go-type game, namely the extended Go(k)-game, or eGo(k)-game for short. This new game is played on oriented graphs.

Let $\vec{G}$ be an oriented graph with $V(\vec{G})=M(\vec{G}) \cup U(\vec{G})$. For every unmarked vertex $u \in U(\vec{G})$, we denote by $N_{M}(u)$ the set of marked neighbours of $u$ (as before) and by $E_{M}(u)$ the set of marked vertices $w$ such that $(i) u$ and $w$ are linked by a directed 2-path 
(in either direction), and (ii) there exists no directed 2-path linking $u$ and $w$ (in either direction) whose middle vertex is marked. (In the following, vertices of $E_{M}(u)$ will be referred to as marked 2-neighbours of $u$.) The extended score of $u$, denoted $s^{*}(u)$, is defined as the sum $s^{*}(u)=\left|N_{M}(u)\right|+\left|E_{M}(u)\right|$ (see Figure 1, where marked vertices are drawn as black vertices). The extended score of the graph $\vec{G}$, denoted by $s^{*}(\vec{G})$, is then defined as $s^{*}(\vec{G})=\operatorname{Max}\left\{s^{*}(u): u \in U(\vec{G})\right\}$ if $U(\vec{G})$ is non-empty and $s^{*}(\vec{G})=0$ otherwise.

The two-players games $e G o(k)$ is defined as follows. Alice and Bob play alternatively with Alice having the first move. A move consists in marking any unmarked vertex. The game thus finishes after $n$ moves, when all vertices are marked. Alice wins the game if after each move (of any player) the extended score of the current graph is at most $k$.

The extended Go-number (or eGo-number for short) of $\vec{G}$, denoted by $e G o(\vec{G})$, is then defined as the least $k$ such that Alice has a winning strategy in the game $e G o(k)$. Clearly, for every orientation $\vec{G}$ of an undirected graph $G$ the inequality $G o(G) \leq e G o(\vec{G})$ holds. Moreover, for every orientation $\vec{G}$ we have $e G o(\vec{G}) \leq \Delta(\vec{G})+\Delta(\vec{G})(\Delta(\overrightarrow{\vec{G}})-1)=\Delta^{2}(\vec{G})$.

We now introduce a special type of tournaments that we shall use as target graphs for defining a winning strategy for Alice when playing the oriented coloring game on graphs with bounded eGo-number.

Let us call orientation vector of length $k$ a $k$-tuple $\nu=\left(\nu_{1}, \nu_{2}, \ldots, \nu_{k}\right) \in\{0,1\}^{k}$. If $\vec{G}$ is an oriented graph and $S=\left(u_{1}, u_{2}, \ldots, u_{k}\right)$ a sequence of $k$ distinct vertices of $V(\vec{G})$, we say that a vertex $v \in V(\vec{G})$ is a $\nu$-successor of $S$ if, for every $i, 1 \leq i \leq k, u_{i} v \in E(\vec{G})$ if $\nu_{i}=1$ and $v u_{i} \in E(\vec{G})$ otherwise.

Definition 2 (Property $P(k)$ ) We say that a tournament $\vec{T}$ satisfies the property $P(k)$ if $(i) \vec{T}$ has at least $k$ vertices and (ii) for every sequence $S=\left(t_{1}, t_{2}, \ldots, t_{k}\right)$ of $k$ distinct vertices in $V(\vec{T})$ and every orientation vector $\nu$ of length $k$, the sequence $S$ has a $\nu$ successor in $\vec{T}$.

The following observation will be later useful:

Observation 3 If $\vec{T}$ is a tournament satisfying the property $P(k)$ then for every sequence $S=\left(t_{1}, t_{2}, \ldots, t_{k^{\prime}}\right)$ of $k^{\prime} \leq k$ distinct vertices in $V(\vec{T})$ and every orientation vector $\nu$ of length $k^{\prime}$, the sequence $S$ has at least $2^{k-k^{\prime}} \nu$-successors in $\vec{T}$.

This is obvious since any orientation vector of length $k^{\prime}$ can be extended to $2^{k-k^{\prime}}$ disctinct orientation vectors of length $k$.

Schütte and Erdös (see [6] or [13], pp. 28-31) considered a similar property of tournaments, the so-called property $S(k)$, by only requiring the existence of a $\nu$-successor for the orientation vector $\nu=(1,1, \ldots, 1)$. Erdös proved in [6] that for every $k$, there exist finite tournaments satisfying the property $S(k)$. In fact, by slightly modifying Erdös's proof, we get the following

Theorem 4 For every $k>0$, there exists a finite tournament $T_{k}$ satisfying the property $P(k)$. 
Proof. Let $n$ be such that $2^{k}\left(\begin{array}{l}n \\ k\end{array}\right)\left(1-2^{-k}\right)^{n-k}<1$ and consider a random tournament on the set $V=\{1,2, \ldots, n\}$. (By a random tournament we mean here a tournament for which the direction of each arc is determined by the flip of a fair coin.) For every subset $S$ of $k$ distinct vertices and every orientation vector $\nu$ of length $k$, let $A_{S, \nu}$ be the event that there is no $\nu$-successor of $S$ ( $S$ is considered here as the sequence obtained by taking the vertices in increasing order).

Since for each fixed vertex $v \in V \backslash S$, the probability that $v$ is not a $\nu$-successor of $S$ is $1-2^{-k}$ and all these $n-k$ events corresponding to the possible choices of $v$ are independent, we get $\operatorname{Pr}\left(A_{S, \nu}\right)=\left(1-2^{-k}\right)^{n-k}$.

It follows that

$$
\operatorname{Pr}\left(\cup_{S, \nu} A_{S, \nu}\right) \leq \sum_{S, \nu} \operatorname{Pr}\left(A_{S, \nu}\right)=2^{k}\left(\begin{array}{l}
n \\
k
\end{array}\right)\left(1-2^{-k}\right)^{n-k}<1 .
$$

Therefore, with positive probability, there is a tournament on $n$ vertices which satisfies the property $P(k)$.

For small values of $k$, optimal tournaments are known. Let $q$ be a prime number such that $q \equiv 3(\bmod 4)$; the tournament $\overrightarrow{Q R_{q}}$ is then defined by $V\left(\overrightarrow{Q R}_{q}\right)=\{0,1, \ldots, q-1\}$ and for every $u, v \in V(\overrightarrow{Q R}), u v \in E\left(\overrightarrow{Q R_{q}}\right)$ if and only if $v-u(\bmod q)$ is a non-zero quadratic residue of $q$. It can be checked that the tournament $\overrightarrow{Q R_{3}}$, that is the directed cycle on three vertices, satisfies the property $P(1)$, that the tournament $\overrightarrow{Q R}$ satisfies the property $P(2)$, that the tournament $\overrightarrow{Q R}_{19}$ satisfies the property $P(3)$ and that these three solutions are optimal (see [16]).

We are now able to prove the following

Theorem 5 For every positive integer $k$ there exists a number $c(k)$ such that for every oriented graph $\vec{G}$, if eGo( $\vec{G}) \leq k$ then $\operatorname{ogcn}(\vec{G}) \leq c(k)$.

Proof. We know that Alice has a winning strategy for the game $e G o(k)$ on $\vec{G}$. Let $\vec{T}$ be a finite tournament satisfying the property $P(k)$ (we know by Theorem 4 that such a tournament exists) and let $c(k)$ denote the order of $\vec{T}$. We claim that if Alice uses the same strategy (for choosing the vertex to be colored) when playing the oriented coloring game on $\vec{G}$ with $\vec{T}$ as a target graph then the whole graph can be $\vec{T}$-colored and Alice wins the game. Since $\vec{T}$ has $c(k)$ vertices, this will prove the desired result.

To see that, recall that playing the $e G o(k)$-game strategy ensures that after each move every uncolored vertex $v$ has at most $k_{1}$ colored neighbours and $k_{2}$ colored 2-neighbours with $k_{1}+k_{2} \leq k$. The property $P(k)$ then ensures that such a vertex $v$ can always be $\vec{T}$-colored: if $c_{1}, c_{2}, \ldots, c_{k_{1}}$ denote the colors of the $k_{1}$ colored neighbours of $v$, we know that there exist $2^{k-k_{1}}>k_{2}$ colors in $\vec{T}$ available for $v$ according to the direction of the arcs linking $v$ to its $k_{1}$ colored neighbours. Let $\alpha$ be such a color. The color $\alpha$ cannot be used for $v$ if and only if there exists a vertex $w$ in $\vec{G}$, already colored with $\alpha$, such that $v$ and $w$ are linked by a directed path of length 2 (in either direction). Moreover, $v$ and $w$ are not linked by some directed path of length 2 whose middle vertex $x$ is already colored 
by some $c_{i}, 1 \leq i \leq k_{1}$, since in that case $\alpha c_{i}$ and $c_{i} \alpha$ would be two opposite arcs in $\vec{T}$. Therefore, the vertex $w$ is a colored 2-neighbour of $v$. Since $v$ has at most $k_{2}$ such colored 2-neighbours, there is still at least one color available for $v$.

Since for every oriented graph $\vec{G}$ we have $e G o(\vec{G}) \leq \Delta^{2}(\vec{G})$, we get that the oriented game chromatic number of every oriented graph $\vec{G}$ is bounded by some constant only depending on $\Delta(\vec{G})$. The oriented game chromatic number is thus a well-defined parameter. However, compare this with a far less obvious problem discussed in the last section.

\section{Paths, cycles and trees}

We study in this section the oriented game chromatic number of oriented paths, oriented cycles and oriented trees.

Since every vertex in a path or a cycle has degree at most 2 the eGo-number of paths or cycles is obviously at most 2 . Using the tournament $\overrightarrow{Q R}$ introduced in the previous section, which satisfies the property $P(2)$, we get:

Theorem 6 If $\vec{G}$ is an oriented path or an oriented cycle, then ogcn $(\vec{G}) \leq 7$.

In fact, this bound is tight, as shown by the following:

Theorem 7 There exist oriented paths with oriented game chromatic number 7.

Proof. The idea is to prove that for a specific path, Alice cannot win the game if she uses a target graph which does not satisfy the property $P(2)$. Since no graph having less than 7 vertices satisfies this property, we get the result.

Therefore, suppose that the game is played with a target graph $\vec{H}$ which does not satisfy the property $P(2)$. It means that there exist two distinct vertices in $\vec{H}$, say $a$ and $b$, and an orientation vector $\nu$ of size 2 , such that the sequence $(a, b)$ has no $\nu$-successor in $\vec{H}$. We claim that there exists an oriented path $\vec{P}$ such that Bob has a winning strategy when playing the oriented coloring game on $\vec{P}$.

Let $\vec{P}=x_{1} y_{1} z_{1} t_{1} u_{1} v x_{2} y_{2} z_{2} t_{2} u_{2}$ be the path on 10 vertices oriented in such a way that $y_{i}$ is a $\nu$-successor in $\vec{P}$ of $\left(z_{i}, x_{i}\right)$ and $t_{i}$ is a $\nu$-successor in $\vec{P}$ of $\left(z_{i}, u_{i}\right)$ for every $i \in\{1,2\}$. Bob's strategy is the following: after Alice's first move, Bob chooses the vertex $z_{i}, i \in\{1,2\}$, such that none of $x_{i}, y_{i}, z_{i}, t_{i}, u_{i}$ have been colored, and colors it with $a$. On his second turn, Bob chooses either the vertex $x_{i}$, if none of $x_{i}, y_{i}$ have been colored, or the vertex $u_{i}$ otherwise, and colors it with $b$. Clearly, the path $\vec{P}$ can no longer be $\vec{H}$-colored.

Faigle, Kern, Kierstead and Trotter proved in [7] that the game chromatic number of every tree is at most 4 (Bodlaender gave in [1] examples of trees with game chromatic number 4). In fact they implicitely deal with the Go-number of trees. The proof we give below first appeared in a manuscript of Kierstead and Tuza and was independtly discovered by Zhu. 
Theorem 8 (Faigle, Kern, Kierstead and Trotter, 1993) If $T$ is an undirected tree then $G o(T) \leq 3$.

Proof. We shall give a winning strategy for Alice when playing the game Go(3). During the whole game, Alice will ensure that every subtree $T^{\prime}$ of $T$ only containing unmarked vertices is adjacent to at most two marked vertices. On her first move, Alice marks any vertex and this condition obviously holds. Suppose now it holds before a move of Bob. After Bob's move, at most one unmarked subtree is adjacent to three marked vertices. Alice then marks the unique vertex which lies on the three paths linking pairs of these marked vertices. After this move, again the condition holds.

In fact, the strategy used in this proof even ensures that if the eGo-game is played on an oriented tree the extended score of every intermediate tree is at most 3 and we get:

Theorem 9 If $\vec{T}$ is an oriented tree then eGo $(\vec{T}) \leq 3$.

From Theorems 5 and 9, we know that the oriented game chromatic number of any oriented tree is bounded by the order of any tournament satisfying the property $P(3)$. Using the tournament $\overrightarrow{Q R}$ in introduced in the previous section, we get:

Theorem 10 If $\vec{T}$ is an oriented tree, then $\operatorname{ogcn}(\vec{T}) \leq 19$.

\section{Outerplanar graphs}

It is not difficult to observe that every outerplanar graph has acyclic chromatic number at most 3 (see e.g. [16]). Using the result of Dinski and Zhu [5] we then get that the game chromatic number of every outerplanar graph is at most 12. In [8], Guan and Zhu reduced this bound to 7 . Kierstead and Trotter exhibited in [11] an outerplanar graph with game chromatic number 6 . The question whether there exist outerplanar graphs with game chromatic number 7 or not is still an open question.

We shall prove in this section that the oriented game chromatic number of oriented outerplanar graphs is also bounded. Considering the eGo-game on outerplanar graphs we have the following:

Theorem 11 If $\vec{G}$ is an oriented outerplanar graph then eGo $(\vec{G}) \leq 29$.

Proof. Since any winning strategy on a graph is also a winning strategy on each of its subgraphs we may assume without loss of generality that the graph $\vec{G}$ is maximal outerplanar. In that case, each face of $\vec{G}$ except the infinite face is a triangle.

We first discuss some structural properties of $\vec{G}$. Since these properties are independent from the orientation of $\vec{G}$, we shall say that $u v$ is an edge in $\vec{G}$ whenever $u v$ or $v u$ is an arc in $\vec{G}$.

We consider a linear ordering $v_{1}, v_{2}, \ldots, v_{n}$ of the vertices of $\vec{G}$ such that $v_{1} v_{2}$ is an edge (that is an arc in either direction) and every vertex $v_{i}, i>2$, has exactly two neighbours 


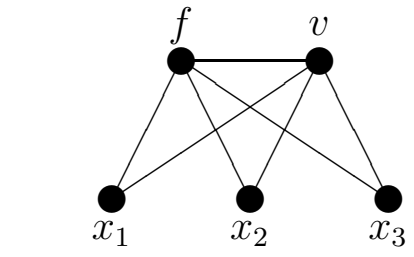

$f\left(x_{1}\right)=f\left(x_{2}\right)=f\left(x_{3}\right)=f$

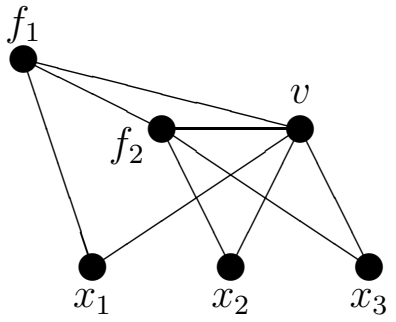

$f\left(x_{1}\right)=f_{1}, \quad f\left(x_{2}\right)=f\left(x_{3}\right)=f_{2}$

Figure 2: Every vertex $v$ has at most 2 nephews

$v_{i_{1}}$ and $v_{i_{2}}$ such that $i_{1}<i_{2}<i$. (Such an ordering exists since $\vec{G}$ is a 2-tree.) For simplicity, we shall write $x<y$ if $x$ and $y$ are two vertices with $x=v_{i}, y=v_{j}$ and $i<j$.

For every vertex $v \notin\left\{v_{1}, v_{2}\right\}$, the father of $v$, denoted by $f(v)$, is the smallest neighbour of $v$ and the uncle of $v$, denoted by $u(v)$, is the smallest neighbour of $v$ distinct from $f(v)$ (we thus have $f(v)<u(v)<v$ and $v$ has no other smaller neighbour). Moreover, we say that $v_{1}$ is the father of $v_{2}$ and that $v$ is a nephew of $u(v)$.

Since $\vec{G}$ is maximal outerplanar, we have:

Claim 1. For every vertex $v \notin\left\{v_{1}, v_{2}\right\}, f(v) u(v)$ is an edge in $\vec{G}$.

Considering the number of nephews that a vertex can have, we have:

Claim 2. Every vertex $v$ has at most 2 distinct nephews.

To see that, suppose that $v$ has 3 distinct nephews, say $x_{1}, x_{2}$ and $x_{3}$. For every $i, 1 \leq i \leq 3, f\left(x_{i}\right)<v$ and $f\left(x_{i}\right) v$ is an edge. The set $\left\{f\left(x_{1}\right), f\left(x_{2}\right), f\left(x_{3}\right)\right\}$ has thus cardinality 1 or 2. Therefore, $\vec{G}$ contains $K_{2,3}$ or a subdivision of $K_{2,3}$ as a subgraph, which contradicts the fact that $\vec{G}$ is outerplanar (see Figure 2).

We call a strong edge an edge linking a vertex and its father. An edge which is not strong is a weak edge. Then we have:

Claim 3. The subgraph $\vec{T}(\vec{G})$ induced by the strong edges is a spanning tree of $\vec{G}$.

To see that, observe that $f(v)$ is defined for every vertex $v \neq v_{1}$ and is such that $f(v)<v$. Therefore, the graph $\vec{T}(\vec{G})$ has $n-1$ edges and is acyclic (in the undirected sense).

Suppose now that Alice plays the eGo(29)-game on $\vec{G}$ as if she were playing on the subgraph $\vec{T}(\vec{G})$, using the strategy defined in the proof of Theorem 8. We claim that this strategy is a winning strategy for the eGo(29)-game on $\vec{G}$.

At each step of the game, each unmarked vertex $v$ has at most 3 marked neighbours in $\vec{T}(\vec{G})$ and, by Claim 2, at most 3 marked neighbours not in $\vec{T}(\vec{G})$ (at most one uncle and two nephews). Therefore, the score $s(v)$ of $v$ is at most 6 .

Let us now consider the marked 2-neighbours of $v$. The vertex $v$ has at most one father, one uncle and two nephews, each of them having at most 5 marked neighbours. (As discussed above, they have at most 6 marked neighbours, but including $v$ which is 
unmarked). In order to prove that the number of marked 2-neighbours of $v$ is bounded, we thus only have to consider the marked neighbours of the sons of $v$. There are at most 3 such marked sons, while the number of unmarked sons is unbounded.

Let us first consider the at most 3 marked sons of $v$ and let $m$ be such a son. Since $m$ is marked, $m$ can produce a marked 2-neighbour $n$ of $v$ if and only if the path vmn is not a directed 2-path and there exists a directed 2-path $v x n$ linking $v$ and $n$. However, since $\vec{G}$ is outerplanar, $m$ can produce at most 2 such marked 2-neighbours (otherwise $\vec{G}$ would contain a subdivision of $K_{2,3}$ ).

Consider now the unmarked sons of $v$. Due to Alice's strategy, we already know that, altogether, the unmarked sons of $v$ have at most 3 marked neighbours in $\vec{T}(\vec{G})$ (otherwise, the extended score of $v$ in $\vec{T}(\vec{G})$ would be greater than 3 ), already considered as marked 2-neighbours of $v$ in $\vec{T}(\vec{G})$. Therefore, we need to bound the number of marked weak neighbours of the unmarked sons of $v$ (by a weak neighbour we mean a neighbour linked by a weak edge). We claim that all such vertices have already been considered (counted). Recall that every vertex has at most 3 weak neighbours, its uncle and its two nephews and let $s$ be any unmarked son of $v$.

1. The uncle $u(s)$ of $s$ has already been considered since it is a neighbour of $v$.

2. Let $t$ be a nephew of $s$. The father $f(t)$ of $t$ is a neighbour of $s$ with $f(t)<s$. Therefore, either $f(t)=f(s)=v$ or $f(t)=u(s)$. In the former case, $t$ has already been considered as a neighbour of $v$. In the latter case, $t$ has already been considered as a 2-neighbour of $v$ in $\vec{T}(\vec{G})$ (if $f(t)$ is a son of $v$ ) or as a neighbour of a nephew of $v$ otherwise.

Therefore, no unmarked son of $v$ can provide a new marked 2-neighbour of $v$. Finally, each unmarked vertex $v$ has:

- at most 3 marked neighbours or 2-neighbours in $\vec{T}(\vec{G})$,

- at most 1 father and 3 weak neighbours, each of them having at most 5 marked neighbours,

- at most 6 marked 2-neighbours contributed by its at most 3 marked sons,

- no other marked 2-neighbour contributed by its unmarked sons.

The extended score $s^{*}(v)$ of $v$ is thus at most $3+4 \times 5+6=29$. This finishes the proof.

Theorems 5 and 11 lead to the following

Corollary 12 There exists a constant $t>0$ such that ogcn $(\vec{G}) \leq t$ for every oriented outerplanar graph $\vec{G}$. 


\section{Discussion and open problems}

In this paper, we have shown how to use the notion of the eGo-number of a graph in order to derive a bound for the oriented game chromatic number of any of its orientations. It would be interesting to determine the exact bound for the oriented game chromatic number of oriented trees. In the same vein, it seems that our upper bound on the eGonumber of oriented outerplanar graphs can easily be improved by looking carefully to the adjacency constraints. Here again, it would be interesting to derive an exact bound.

In the undirected case, a family of graphs with bounded Go-number has bounded game chromatic number. However, having bounded Go-number is not a necessary condition for a family of graphs for having bounded game chromatic number. For instance, it is easy to see that any bipartite complete graph $K_{n, n}, n>1$, has game chromatic number 3 while its Go-number is $n$. In the oriented case, it is not so clear whether having bounded eGonumber is a necessary condition for having bounded oriented game chromatic number or not.

It seems not so easy to prove (or disprove) that the eGo-number of planar graphs or of partial $k$-trees is bounded (Zhu proved in [18] that the game chromatic number of every partial $k$-tree is at most $3 k+2)$. We thus propose the two following problems:

Problem 1 Does there exist a constant $t$ such that every oriented planar graph has oriented game chromatic number at most $t$ ?

Problem 2 Let $k>1$ be an integer. Does there exist a constant $t_{k}$ such that every oriented partial $k$-tree has oriented game chromatic number at most $t$ ?

In our definition of the oriented version of the coloring game, we have used a given oriented graph $\vec{H}$ as a target graph (in other words, Alice's goal is to build a $\vec{H}$-coloring of the graph on which the game is played). Another possibility is to define the oriented coloring game by giving the maximum number of colours that can be used but without choosing a fixed target graph. In that case, the target is built while the game is played, each move having to be "compatible" with the target under construction.

In the undirected case, the coloring game corresponds to this second version. However, fixing the maximum number $k$ of colours that can be used is clearly equivalent to playing with the complete graph $K_{k}$ as a target graph. The situation is much less clear in the oriented case and we do not know whether families of graphs with bounded oriented game chromatic number (with fixed target) and families of graphs with bounded oriented game chromatic number (with fixed maximum number of colours) coincide or not.

Remark. We have been kindly informed by Kierstead [10] about his related results with Trotter and with Tuza (unpublished). They define the complete Go-number (cGonumber) of a graph as the least $k$ so that when Alice and Bob play the Go-game Alice can prevent any unmarked vertex from being connected to more than $k$ marked vertices by paths whose internal vertices are all unmarked. Then, for every orientation $\vec{G}$ of a graph $G, G o(G) \leq e G o(\vec{G}) \leq c G o(G)$. Kierstead and Trotter proved that if $C$ is a class of graphs closed under topological minors that does not contain a $k$-clique for some $k$ 
then the cGo-number of $C$ is bounded by a function of $k$. In particular, planar graphs form such a class. Although they did not considered oriented game chromatic number, (defined in this paper), via our Theorem 5, this gives a positive answer to our Problem 1. Kierstead and Tuza proved that if $G$ is a chordal graph then $c G o(G) \leq 6 w-9$, where $w$ is the clique number of $G$. This allows to decrease to 9 the bound we gave in Theorem 11 for outerplanar graphs and, moreover, gives a positive answer to our Problem 2.

\section{References}

[1] H.L. Boadlaender, On the complexity of some coloring games, Internat. J. Found. Comput. Sci. 2 (1991), 133-147.

[2] H.L. Bodlaender and D. Kratsch, The complexity of coloring games on perfect graphs, Theoret. Comput. Sci. 106 (1992), 309-326.

[3] O. Borodin, On acyclic colorings of planar graphs, Discrete Math. 25 (1979), 211-236.

[4] L. Cai and X. Zhu, Game chromatic index of k-degenerate graphs, manuscript (1998).

[5] T. Dinski and X. Zhu, Game chromatic number of graphs, Discrete Math. 196 (1999), 109-115.

[6] P. Erdös, On a problem in graph theory, Math. Gaz. 47 (1963), 220-223.

[7] U. Faigle, U. Kern, H.A. Kierstead and W.T. Trotter, On the game chromatic number of some classes of graphs, Ars Combinatoria 35 (1993), 143-150.

[8] D. Guan and X. Zhu, The game chromatic number of outerplanar graphs, J. Graph Theory 30 (1999), 67-70.

[9] H.A. Kierstead, A simple competitive graph coloring algorithm, J. Combin. Theory Ser. $B(2000), 57-68$.

[10] H.A. Kierstead, personal communication.

[11] H.A. Kierstead and W.T. Trotter, Planar graph coloring with an uncooperative partner, J. Graph Theory 18-6 (1994), 569-584.

[12] P.C.B. Lam, W.C. Shiu and B. Xu, Edge game coloring of graphs, preprint (1999).

[13] J.W. Moon, Topics on tournaments, Holt, Rinehart and Winston, New York (1968).

[14] J. Nešetřil and E. Sopena, On four coloring problems, KAM-DIMATIA Series 99418, Charles University, Prague.

[15] A. Raspaud and E. Sopena, Good and semi-strong colorings of oriented planar graphs, Inform. Processing Letters 51 (1994), 171-174. 
[16] E. Sopena, On the chromatic number of oriented graphs, J. Graph Theory 25 (1997), 191-205.

[17] X. Zhu, The game coloring number of planar graphs, J. Combin. Theory Ser. B 75 (1999), 245-258.

[18] X. Zhu, The game coloring number of pseudo partial $k$-trees, manuscript (1999). 